Fukushima, M.

Osaka J. Math.

23 (1986), 69-75

\title{
ON THE CONTINUITY OF PLURISUBHARMONIC FUNCTIONS ALONG CONFORMAL DIFFUSIONS
}

\author{
Masatoshi FUKUSHIMA
}

(Received November 8, 1984)

\section{Introduction}

A stochastic process $Z_{t}=\left(Z_{t}^{1}, \cdots, Z_{t}^{n}\right)$ taking values in $C^{n}$ is called a conformal martingale if $Z_{t}^{\alpha}$ and $Z_{t}^{\alpha} Z_{t}^{\beta}, 1 \leqq \alpha, \beta \leqq n$, are continuous local martingales. When $Z_{t}$ is defined only on a time interval $[0, \eta)$ for some predictable stopping time $\eta, Z_{t}$ is said to be a conformal martingale if so is the stopped process $Z_{t \wedge \eta^{\prime}}$ for any stopping time $\eta^{\prime}$ strictly less than $\eta$.

Let $M$ be a complex manifold of complex dimension $n$. By a diffusion process $\boldsymbol{D}=\left(Z_{t}, P_{z}\right)$ on $M$, we mean a strong Markov process on $M$ with continuous sample paths defined on $[0, \zeta), \zeta$ being the life time. In this paper, we assume without specific mention that the diffusion $\boldsymbol{D}$ admits no killing inside $M$ in the sense that $P_{z}\left(\tau_{U}<\zeta<+\infty\right)=P_{z}(\zeta<+\infty), z \in U$, for any relatively compact open set $U \subset M$, where $\tau_{U}$ denotes the first exit time from $U: \tau_{U}=$ inf $\left\{t \geqq 0: Z_{t} \notin U\right\}$. We see then that, for any open set $U \subset M, \tau_{U}$ is a predictable stopping time with respect to $P_{z}$ for $z \in U$.

We call a diffusion process $\boldsymbol{D}=\left(Z_{t}, P_{z}\right)$ on $M$ a conformal diffusion on $M$ if, for any holomorphic coordinate neighbourhood $(U, \phi)$, the $C^{n}$-valued process $\phi\left(Z_{t}\right)$ defined on $\left[0, \tau_{U}\right)$ is a conformal martingale with respect to $P_{z}$ for each $z \in U$. We occasionally assume that the transition function $p_{t}$ of $\boldsymbol{D}$ is absolutely continuous with respect to a volume element $V$ on $M$ :

$$
p_{t}(z, \cdot)<V, \quad z \in M \text {. }
$$

We aim at proving the following theorem.

Theorem. Let $\boldsymbol{D}=\left(Z_{t}, P_{z}\right)$ be a conformal diffusion on $M$ satisfying the condition (1.1). Then, for any plurisubharmonic function $u$ on $M$,

$$
P_{z}\left(u\left(Z_{t}\right) \text { is continuous in } t \in[0, \zeta) \text { and finite for } t \in(0, \zeta)\right)=1, z \in M \text {. }
$$

This is a generalization of a theorem of Doob [2] to the cases of higher complex dimension and our proof is also similar to the one given in [2] in the sense that we utilize the quasi-continuity of plurisubharmonic functions with respect to a specific capacity related to the extremal function. 
As we shall see, any plurisubharmonic function $u$ on $M$ is $D$-subharmonic in Dynkin's sense and consequently $u\left(Z_{t}\right)$ is right continuous. Therefore its continuity would follow from a Hunt's theorem on the regularity of excessive functions provided that

every semi-polar set is polar

for the diffusion $\boldsymbol{D}$. However, it seems to be unknown whether (1.2) is fulfilled for all the conformal diffusions being considered.

Indeed, a typical conformal diffusion is a diffusion $\boldsymbol{D}$ on $M$ whose infinitesimal generator is expressible on a local chart as

$$
L=\frac{1}{2} \sum g^{\alpha \bar{\beta}} \frac{\partial^{2}}{\partial z^{\alpha} \partial \bar{z}^{\beta}}
$$

with a continuous non-negative Hermitian tensor field $g$ on $M$ ([6]). If $g$ is sufficiently smooth and non-degenerate, (1.2) is known to be correct for $\boldsymbol{D}$. But, if $g$ is merely continuous positive or degenerate ( $\boldsymbol{D}$ may still satisfy (1.1) in the latter case), we do not know to what extent (1.2) is true. (1.2) becomes true under the additional condition of the symmetrizability. But the latter condition might be false either in general in view of a Fujita's result [4] saying that there exists a manifold $M$ where no diffusion with generator (1.3) is symmetrizable (although only smooth and non-degenerate cases are treated in [4]).

We add a remark that there are many conformal diffusions whose generators are not expressible by the usual differential operator like (1.3). It was shown in [5] that fairly general class of symmetrizable conformal diffusions can be characterized by closed positive currents of type $(n-1, n-1)$. The first two propositions of the present paper have been proven in [5] for this class of diffusions on a domain of $C^{n}$.

This work was motivated by the lectures of Professor Laurent Schwartz delivered at Kyoto University (cf. [7]). I am grateful to him for his kind guidance to the problem.

\section{D-subharmonicity of plurisubharmonic functions}

A function $u$ on an open set $E \subset M$ taking values in $[-\infty,+\infty)$ is said to be plurisubharmonic on $E$ if, on each holomorphic coordinate neighbourhood $U \subset E, u$ is locally integrable, $\sum \frac{\partial^{2} u}{\partial z^{\alpha} \partial \bar{z}^{\beta}} \xi^{\alpha} \xi^{\beta}$ is a non-negative distribution for any $\xi=\left(\xi^{1}, \cdots, \xi^{n}\right) \in C^{n}$ and ess $\lim _{z^{\prime} \rightarrow z} \sup u\left(z^{\prime}\right)=u(z), z \in U$. Those properties are intrinsic because they are preserved under holomorphic transformations. Any plurisubharmonic function is upper semicontinuous.

Let $\boldsymbol{D}=\left(Z_{t}, P_{z}\right)$ be a conformal diffusion on $M$ with transition function $p_{t}$. 
A Borel function $u$ on $M$ taking values in $[0,+\infty]$ is called $p_{t}$-excessive if $p_{t} u(Z) \uparrow u(z)$ as $t \downarrow 0$ for each $z \in M$. A Borel function $u$ on an open set $E \subset M$ taking values in $[-\infty,+\infty)$ is called $\boldsymbol{D}$-subharmonic on $E$ if $u$ is $\boldsymbol{D}$-finely upper semicontinuous, locally bounded from above and, for any open set $V$ with compact closure $\nabla \subset E$,

$$
u(z) \leqq E_{z}\left(u\left(Z_{\tau_{F}}\right) ; \tau_{V}<\zeta\right), \quad z \in V .
$$

Any $\boldsymbol{D}$-subharmonic function is $\boldsymbol{D}$-finely continuous and hence right continuous along $Z_{t}$ in $t \in[0, \zeta) P_{z}$-almost surely ([3]). The negative of a $\boldsymbol{D}$-subharmonic function is said to be $\boldsymbol{D}$-superharmonic. A Dynkin's theorem [3; Theorem 12.4] says that a non-negative Borel function on $M$ is $p_{t}$-excessive if and only if it is $\boldsymbol{D}$-superharmonic.

Proposition 1. Any plurisubharmonic function on $M$ is $D$-subharmonic. The negative of a non-positive plurisubharmonic function on $M$ is $p_{t}$-excessive.

Proof. Let $u$ be a plurisubharmonic function on $M$. $u$ is then locally bounded from above on each coordinate neighbourhood. Besides the subharmonicity for the diffusion process is a local property according to a Sur's theorem ([3; Theorem 12.11]). Hence we may only prove the $\boldsymbol{D}$-subharmonicity of $u$ by assuming that $M$ is a bounded domain $D \subset C^{n}$ and $u$ is non-positive.

Take any open set $V$ with compact closure in $D$ and denote by $\tau$ the first exit time of $Z_{t}$ from $V$. Since $\left(Z_{t}, P_{z}\right)$ is a conformal martingale, we see, by virtue of Schwartz [6; Proposition (5.10)], that $\left(u\left(Z_{t \wedge \tau}\right), P_{z}\right)$ is a generalized submartingale for $z \in V$, and consequently,

$$
-\infty \leqq u(z) \leqq E_{z}\left(u\left(Z_{t \wedge \tau}\right)\right), \quad z \in V .
$$

The right hand side is not greater than $E_{z}\left(u\left(Z_{t \wedge \tau}\right) ; \tau<\zeta\right)$ and we get the inequality (2.1) by letting $t \rightarrow+\infty$. Since $u$ is upper semicontinuous, we conclude that $u$ is $\boldsymbol{D}$-subharmonic.

In the remainder of this section, we only consider a bounded domain $D$ of $C^{n}$. For $E \subset D$, the extremal function $u_{E}^{*}$ is defined by $u_{E}(z)=\sup \{v(z): v$ plurisubharmonic on $D,-1 \leqq v \leqq 0$ on $D, v=-1$ on $E\}, u_{E}^{*}(z)=\varlimsup_{z^{\prime} \rightarrow z} u_{E}\left(z^{\prime}\right)$, $z \in D$. We further introduce a set function $C_{z}$ by

$$
C_{\sharp}(E)=-\int_{D} u_{E}^{*}(z) d V(z) \quad\left(=-\int_{D} u_{E}(z) d V(z)\right)
$$

where $V$ denotes the Lebesgue measure on $D . \quad C_{\ddagger}$ is known to be a Choquet capacity ([1; Proposition 8.4]). Moreover $C_{\xi}(N)=0$ if and only if $N$ is pluripolar, namely, there exists a plurisubharmonic function $v$ on $D$ with $N \subset v^{-1}(-\infty)$.

Let $\boldsymbol{D}=\left(Z_{t}, P_{z}\right)$ be a conformal diffusion on $D$. Denote by $\sigma_{E}$ the hitting 
time of a set $E \subset D$ after $0+: \sigma_{E}=\inf \left\{t>0: Z_{t} \in E\right\}$. We let $\sigma_{E}=+\infty$ if the event in the braces is empty.

Proposition 2. For any Borel set $E \subset D$,

$$
\int_{D} P_{z}\left(\sigma_{E}<\zeta\right) d V(z) \leqq C_{\sharp}(E)
$$

Proof. By Choquet's lemma, there is a non-decreasing sequence of plurisubharmonic functions $v_{k}$ such that $-1 \leqq v_{k} \leqq 0, v_{k}=-1$ on $E$ and $u_{E}^{*}(z)=$ $\varlimsup_{z^{\prime} \rightarrow z} v_{0}\left(z^{\prime}\right)$ for $v_{0}=\lim _{k \rightarrow \infty} v_{k}$. By Proposition 1, $\left\{-v_{k}\left(Z_{t}\right), P_{z}\right\}$ is supermartingale and $-v_{k}(z) \geqq-E_{z}\left(v_{k}\left(Z_{\sigma_{K}}\right): \sigma_{K}<\zeta\right) \geqq P_{z}\left(\sigma_{K}<\zeta\right)$ for any compact set $K \subset E$ and $z \in D$, on account of the optional sampling theorem. Letting $k \rightarrow \infty$ and integrating by $d V$, we have

$$
\int_{D} P_{z}\left(\sigma_{K}<\zeta\right) d V(z) \leqq C_{\sharp}(E)
$$

since $v_{0}=u_{E}^{*} V$-a.e. Taking then an increasing sequence of compact sets $K_{m} \subset E$ such that $\sigma_{K_{m}} \downarrow \sigma_{E}$ as $m \rightarrow \infty, P_{V}$-a.e., we get the desired inequality.

\section{Corollary 1}

(i) If $N \subset D$ is pluripolar, then there exists a Borel set $N^{\prime} \supset N$ and

$$
P_{z}\left(\sigma_{N^{\prime}}<\zeta\right)=0 \quad V \text {-a.e. } \quad z \in D .
$$

(ii) If $O_{k} \subset D$ are decreasing open sets such that $\lim _{k \rightarrow \infty} C_{\ddagger}\left(O_{k}\right)=0$, then

$$
P_{z}\left(\lim _{k \rightarrow \infty} \sigma_{O_{k}}<\zeta\right)=0 \quad V \text {-a.e. } \quad z \in D .
$$

Proof. (ii) is a stronger assertion than (i). (ii) is immediate from Proposition 2 .

We denote by $\theta_{s}$ the usual shift operator defined by $Z_{t}\left(\theta_{s} \omega\right)=Z_{s+t}(\omega)$. In particular we have

$$
s+\sigma_{E} \circ \theta_{s}(\omega)=\inf \left\{t>s: Z_{t}(\omega) \in E\right\}, \quad s \geqq 0 .
$$

Corollary 2. Suppose that the transition function $p_{t}$ of $\boldsymbol{D}$ satisfies the absolute continuity condition (1.1).

(i) If $N \subset D$ is pluripolar, then $N$ is D-polar: there exists a Borel set $N^{\prime} \supset N$ and (2.3) holds for every $z \in D$.

(ii) If $O_{k} \subset D$ are decreasing open sets such that $\lim _{k \rightarrow \infty} C_{\sharp}\left(O_{k}\right)=0$, then

$$
P_{z}\left(\lim _{k \rightarrow \infty}\left(s+\sigma_{O_{k}} \circ \theta_{s}\right)<\zeta, s<\zeta\right)=0
$$

for every $s>0$ and $z \in D$.

Proof. (i) Denote by $f(z)$ the left hand side of $(2.3)$. Then $f(z)=$ 
$\lim _{s \neq 0} p_{s} f(z)=0, z \in D$, by the assumption and Corollary 1 (i). (ii) The left hand side of (2.6) equals $p_{s} f(z)$ for the function $f$ defined by the left hand side of (2.4).

\section{3. $C_{z}$-quasi-continuity of plurisubharmonic functions}

We continue to consider a bounded domain $D \subset C^{n}$ and the capacity $C_{\sharp}$ defined by (2.2).

Proposition 3. Suppose that the domain $D$ is strongly pseudo-convex. Any plurisubharmonic function $u$ on $D$ is then $C_{\sharp}$-quasi-continuous. More specifically, for any $\varepsilon>0$, there exists an open set $O \subset D$ with $C_{\sharp}(O)<\varepsilon$ such that $u$ is finite valued and continuous on $D-O$ with respect to the relative topology.

Proof. We deduce this from several results of Bedford-Taylor [1]. First, according to [1; Theorem 3.5], any plurisubharmonic function on a bounded domain $D$ is quasi-continuous in the above sense but with respect to another capacity which we shall denote by $C_{B T} . \quad C_{B T}$ admits the expression

$$
C_{B T}(O)=\int_{D}\left(d d^{c} u_{0}^{*}\right)^{n}
$$

for open set $O$ with compact closure $\bar{O} \subset D$. Therefore it suffices to show the implication

$$
C_{B T}\left(O_{k}\right) \underset{k \rightarrow \infty}{\rightarrow} 0 \Rightarrow C_{k}\left(O_{k}\right) \underset{k \rightarrow \infty}{\rightarrow} 0
$$

for decreasing sequence of open sets $O_{k} \subset D$.

A function on $D$ is quasi-continuous relative to a capacity if and only if it is so on each open set $E$ with compact closure $\bar{E} \subset D$. Hence, in proving (3.2), we may assume that $O_{1}$ has compact closure $\bar{O}_{1} \subset D$. Set $v=\lim _{k \rightarrow \infty} u_{O_{k}}^{*}$, $v^{*}(z)=\lim _{z^{\prime} \rightarrow z} v\left(z^{\prime}\right)$, and assume now the strong pseudo-convexity of $D$. We then easily see that $v^{*}(z) \rightarrow 0$ as $z \rightarrow \partial D$. Moreover by the continuity of the Bedford-Taylor measures [1; Proposition 5.2], $\left(d d^{c} u_{O_{k}}^{*}\right)^{n} \rightarrow\left(d d^{c} v^{*}\right)^{n}, k \rightarrow \infty$. Hence we get, from (3.1) and the hyposesis in (3.2), $\int_{D}\left(d d^{c} v^{*}\right)^{n}=0$ and consequently $\left(d d^{c} v^{*}\right)^{n}$ is the zero measure. We can finally use a comparison theorem [1, Corollary 4.4] to obtain $v^{*}=0$ and $v=0 V$-a.e. We arrive at the conclusion in (3.2): $\lim _{k \rightarrow \infty} C_{\sharp}\left(O_{k}\right)=\int_{D} v(z) d V(z)=0$.

\section{Proof of Theorem}

The right continuity of $u\left(Z_{t}\right)$ at $t=0$

$$
P_{z}\left(\lim _{t \downarrow 0} u\left(Z_{t}\right)=u(z)\right)=1, \quad z \in M
$$


follows from Proposition 1.

For a stopping time $\eta \in[0,+\infty]$, let us consider the event $\Lambda_{n}=\left\{u\left(Z_{t}\right)\right.$ is finite and continuous for $t \in(0, \eta)\}$. We aim at proving

$$
P_{z}\left(\Lambda_{\zeta}\right)=1, \quad z \in M
$$

We assume the condition (1.1). Choose a system of holomorphic coordinate neighbourhoods $\left(U_{\infty}, \phi_{a}\right)$ of $M$ such that $\phi_{a}\left(U_{\infty}\right)$ is a strongly pseudo-convex bounded domain of $C^{n}$. Proposition 3 and Corollary 2 (ii) to Proposition 2 are applicable to the function $\left.u\right|_{U_{a}}$ and to the part $\boldsymbol{D}_{a}$ of $\boldsymbol{D}$ on $U_{a}$ respectively. In view of (2.5), we then readily see that $P_{z}\left(u\left(Z_{t}\right)\right.$ is finite and continuous for $\left.t \in\left(s, \tau_{U_{\omega}}\right), s^{\prime}<\tau_{U_{\omega}}\right)=P_{z}\left(s^{\prime}<\tau_{U_{\omega}}\right), 0<s \leqq s^{\prime}, z \in U_{\omega}$. By letting $s \downarrow 0$ and then $s^{\prime} \downarrow 0$, we get

$$
P_{z}\left(\Lambda_{\tau \sigma_{\omega}}\right)=1, \quad z \in U_{\alpha} .
$$

We now use a Sur's method. Take two members, say, $U_{0}$ and $U_{1}$ from the chart system and let $V$ be an arbitrary open set with $\bar{V} \subset U_{0} \cup U_{1}$. By [3; Lemma 12.6], we can find open sets $V_{0}$ and $V_{1}$ such that $V=V_{0} \cup V_{1}$, $\bar{V}_{0} \subset U_{0}, \bar{V}_{1} \subset U_{1}$ and $\overline{V_{0} \cap\left(M-V_{1}\right)} \cap \overline{V_{1} \cap\left(M-V_{0}\right)}=\phi$. Denote by $\tau^{0}$ and $\tau^{1}$ the exit time from $V_{0}$ and $V_{1}$ respectively, and let $\gamma_{0}=0, \gamma_{k+1}=\gamma_{k}+\tau^{\varepsilon}{ }_{k \circ} \theta_{\gamma_{k}}$, $k \geqq 1$, where $\varepsilon_{k}=k \bmod 2$. By virtue of [3; Lemma 12.4]; it holds then that

$$
\gamma_{k}=\tau_{V} \quad \text { from some } k \text { on. }
$$

Together with the event $\Lambda_{\eta}$ for the stopping time $\eta$, we also consider the event $\tilde{\Lambda}_{\eta}=\left\{u\left(Z_{t}\right)\right.$ is finite continuous at each $t \in(0, \eta)$ and also at $t=\eta$ if $\left.\eta<+\infty\right\}$. In view of (4.3), we have

$$
P_{z}\left(\tilde{\Lambda}_{\tau^{i}}\right)=1, \quad z \in V_{i}, i=0,1 .
$$

Since (4.5) is trivially true for $z \in D-V_{i}$, we obtain from the strong Markov property and (4.5),

$P_{z}\left(\tilde{\Lambda}_{\gamma_{2}}\right)=P_{z}\left(u\left(Z_{t}\right)\right.$ is finite continuous for $\left.t>0, \tau^{0}=+\infty\right)+E_{z}\left(u\left(Z_{t}\right)\right.$ is finite continuous for $\left.t \in\left(0, \tau^{0}\right], \tau^{0}<+\infty ; P_{z_{\tau} 0}\left(\tilde{\Lambda}_{\tau^{1}}\right)\right)=P_{z}\left(\tilde{\Lambda}_{\tau^{0}}\right)=1, z \in V$. By induction and (4.4), we get

$$
P_{z}\left(\tilde{\Lambda}_{\tau_{\nabla}}\right)=1, \quad z \in V .
$$

By letting $V \downarrow U_{0} \cup U_{1}$, we are led from (4.6) to

$$
P_{z}\left(\Lambda_{\tau_{G}}\right)=1, \quad z \in G,
$$

for $G=U_{0} \cup U_{1}$. Repeating the same argument, (4.7) can be seen to be true for the union $G$ of finite number of $U_{\infty}$ 's. Now (4.7) holds for any relatively compact open set $G \subset M$. We finally let $G \uparrow M$ to get the desired identity (4.2). 


\section{References}

[1] E. Bedford and B.A. Taylor: A new capacity for plurisubharmonic functions, Acta Math. 149 (1982), 1-44.

[2] J.L. Doob: Semimartingales and subharmonic functions, Trans. Amer. Math. Soc. 77 (1954), 86-121.

[3] E.B. Dynkin: Markov processes, Springer, 1965.

[4] T. Fujita: A note on symmetry of conformal diffusions, preprint.

[5] M. Fukushima and M. Okada: On conformal martingale diffusions and pluripolar sets, J. Funct. Anal. 55 (1944), 377-388.

[6] L. Schwartz: Semi-martingales sur des variétés, et martingales conformes sur des variétés analytiques complexes, Lecture Notes in Math., 780, Springer, 1980.

[7] L. Schwartz: Complements sur les martingales conformes, Osaka J. Math. 23 (1986).

Department of Mathematics

College of General Education

Osaka University

Toyonaka, Osaka, Japan 
